\title{
MEASUREMENT OF DRIVER DISTRACTION IN MALAYSIA'S TRAFFIC ENVIRONMENT: A DRIVING SIMULATOR STUDY
}

\section{Mohd Firdaus Mohd Siam*, Mohd Hafzi Md Isa, Nurulhana Borhan, Abdullah Sukardi and Wong Shaw Voon}

\author{
Malaysian Institute of Road Safety Research, \\ 43000 Kajang, Selangor Darul Ehsan, Malaysia \\ Email: mfirdaus@miros.gov.my \\ Phone: +603 8924 9200; Fax: +603 87373994
}

\begin{abstract}
The Malaysian Institute of Road Safety Research has embarked on the development of a fixed-based driving simulator that can be reconfigured easily to suit various road safety research requirements. The objective of this study is to measure driver distraction in terms of participants' response time for different road conditions and secondary tasks using a driving simulator. Three different simulation routes were designed in the study - expressway, off-ramp, and curved road. Thirty participants took part in the study. Two types of detection response task were used in the study - tactile and visual. Recall number, surrogate reference task, navigation, and texting were used as secondary tasks. The results showed that in terms of road segments, both types of detection response task were found to be sensitive; longer response times were observed for more demanding off-ramp and curved road sections when compared with expressway. Furthermore, for secondary tasks, the participants took longer to respond to both stimuli, particularly for the more difficult task followed by an easier task. In general, response times increased as a function of road segments as well as exposure to secondary tasks.
\end{abstract}

Keywords: Driving simulator; driver distraction; response time; secondary task; road safety.

\section{INTRODUCTION}

In 2012, 6,917 fatalities were recorded in Malaysia due to road crashes, with an average of 18 people killed every day [1]. This is not only happening in this country but also worldwide, as road traffic injury is the eleventh leading cause of death, and over one million people are killed every year in road crashes [2]. A previous study showed that human errors are the major contributing factor in approximately $90 \%$ of road traffic accidents [3]; driver distraction is a significant contributor to road traffic accidents [4, 5]. Naturalistic driving studies have demonstrated that drivers have a tendency to spend a vast amount of driving time doing secondary tasks. According to research, approximately $23 \%$ of all crashes and near-crashes were caused by distraction due to secondary tasks [6]. Driving performance begins to deteriorate when drivers fail to allocate sufficient attention to the driving task at critical moments, because they are engaged in another task, thereby resulting in an impairment of the ability to drive safely and effectively [7, 8]. One of the prominent tools to study human-related issues specifically for driver distraction is a driving simulator. It is able to simulate a virtual driving environment and resemble real driving conditions [9-11]. The advantages of 
using a driving simulator as compared to a real road experiment is that the former provides a controlled and repeatable environment, is safer, and less expensive [12]. The objectives of this study are to measure driver distraction in terms of participants' response time in different road conditions and engagement in secondary tasks using a driving simulator. The study was part of international coordinated studies under ISO TC22/SC13/WG8, which aims to support the ongoing development of the new ISO 17488 document [13].

\section{METHODOLOGY}

\section{Participants}

The 30 participants (15 female, 15 male) who participated in this study were all righthanded drivers without any specific knowledge of or expertise in the study. Their ages ranged from 25 to 45 years, with an average of 33.67 years. All participants agreed to participate in the study as volunteers after reading and signing a consent form detailing the purpose and proceedings of the study. They were all licensed drivers and had, on average, driven approximately $27,690 \mathrm{~km}$ a year. The average driving experience of all participants was 10.73 years, and they were able to drive automatic transmission cars. Participants were given RM 200.00 for their full commitment to the study.

\section{Driving Simulator}

The MIROS Driving Simulator Cabin (CabinDS) was used in this study. The platform for the simulator was a second generation Perodua Myvi 1.3L. The main components of the integrated system of CabinDS are steering wheel, pedals, transmission, full car cabin, LCD projector and screen, computer, simulation software, video camera, and sound system. The overview of the integrated system of CabinDS is depicted in Figure 1.

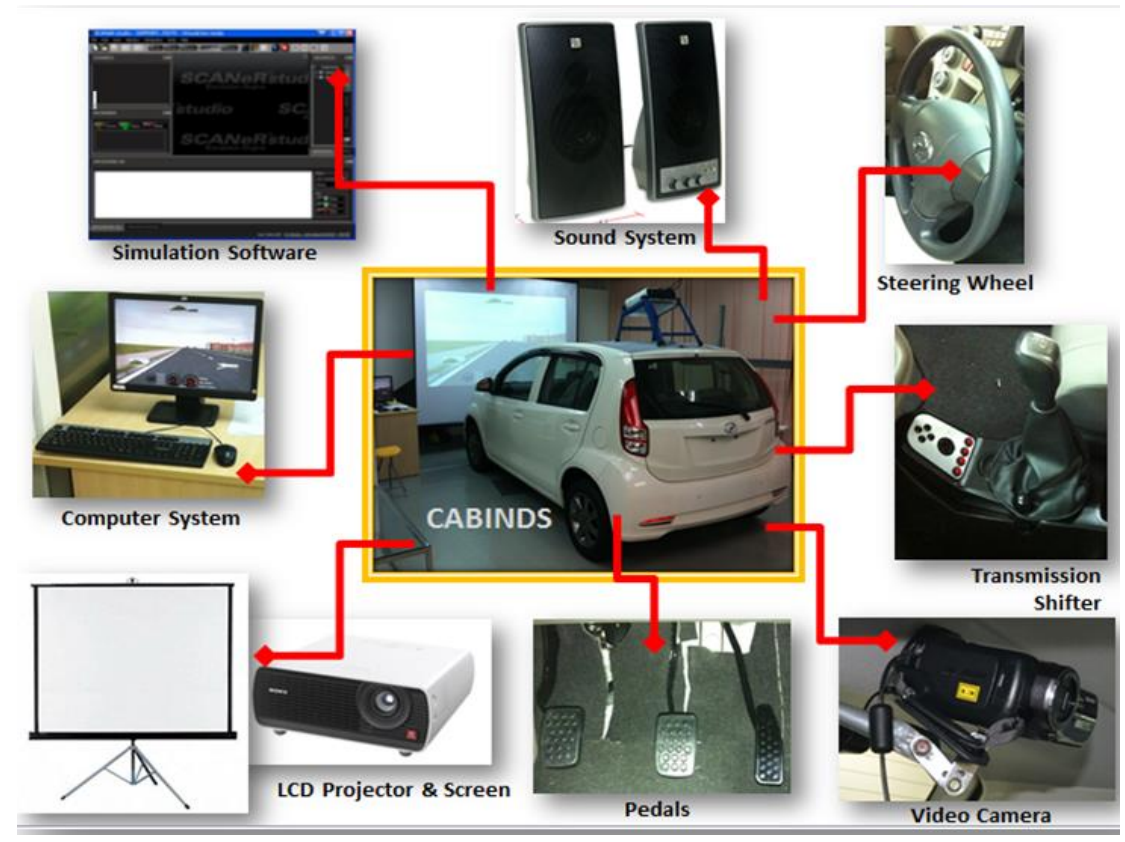

Figure 1. Integrated system of CabinDS. 
Scaner Studio was used as the simulation software in the development of the scenarios. It is a comprehensive driving simulation software package that comprises five main modes, which are Vehicle, Terrain, Scenario, Simulation, and Analysis. Three different roadways were designed in the simulation study - expressway, off-ramp, and curved road. An overview of the designated scenario of the simulation is depicted in Figure 2.

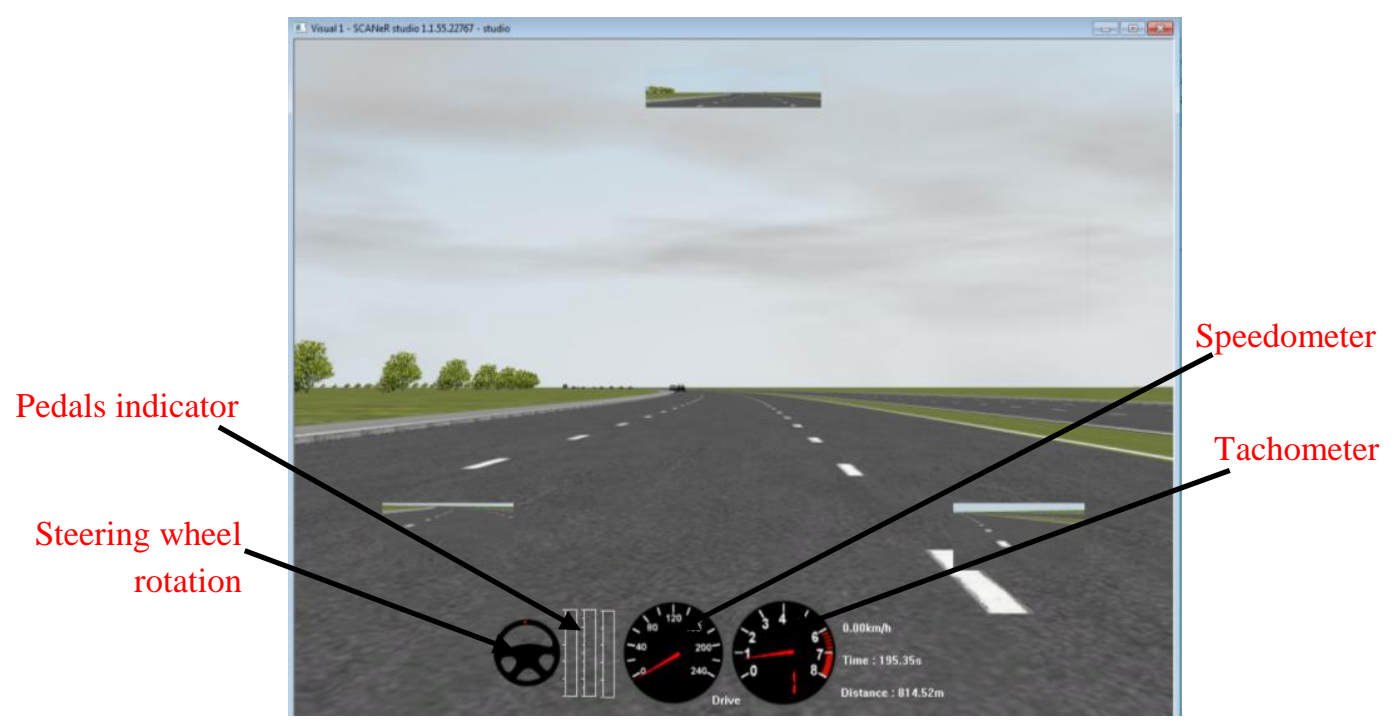

Figure 2. Illustration of the simulation scenario.

\section{Detection Response Task}

Detection Response Task (DRT), previously known as the Peripheral Detection Task, is one of the methods for measuring driver distraction [14]. The task is employed for measuring the effects of the demands of driving and engaging in secondary tasks on driver attention, particularly in the context of driver and vehicle interface evaluation [15]. Two types of DRT were used in the study - tactile and head-mounted. The stimuli were randomly presented at temporal intervals and were uniformly distributed. In the head-mounted type, the visual stimulus was presented by utilizing a single LED attached to the head. For the tactile type, a tactile stimulus was presented by using a small electrical vibrator [16]. The vibrator was placed on the driver's shoulder using medical tape. Participants were required to respond by pressing a microswitch attached to the right index finger [17]. The response was made by pressing the switch to the steering wheel.

\section{Secondary Tasks}

Four secondary tasks were employed in this study - recall number, surrogate reference, navigation, and texting using mobile phone. The Auditory Presentation-Verbal Response Delayed Digit Recall Task (n-back) was used for the secondary tasks. It consists of auditory stimuli that the driver listens to and repeats following the specific rules. The respondents were presented with two levels of difficulty with easy (0-back) and hard (1-back) levels of secondary cognitive workload. The protocols on trainings and the experimental administration of the auditory presentation closely followed that 
suggested by the Massachusetts Institute of Technology (MIT) AgeLab [18]. The surrogate reference task, using the visual task program was used as the secondary task. In this task, circles are presented on a screen and participants use a keypad to choose the section of the screen with the larger circle. Two types of conditions were used: easy and difficult [19]. The task setup is illustrated in Figure 3.

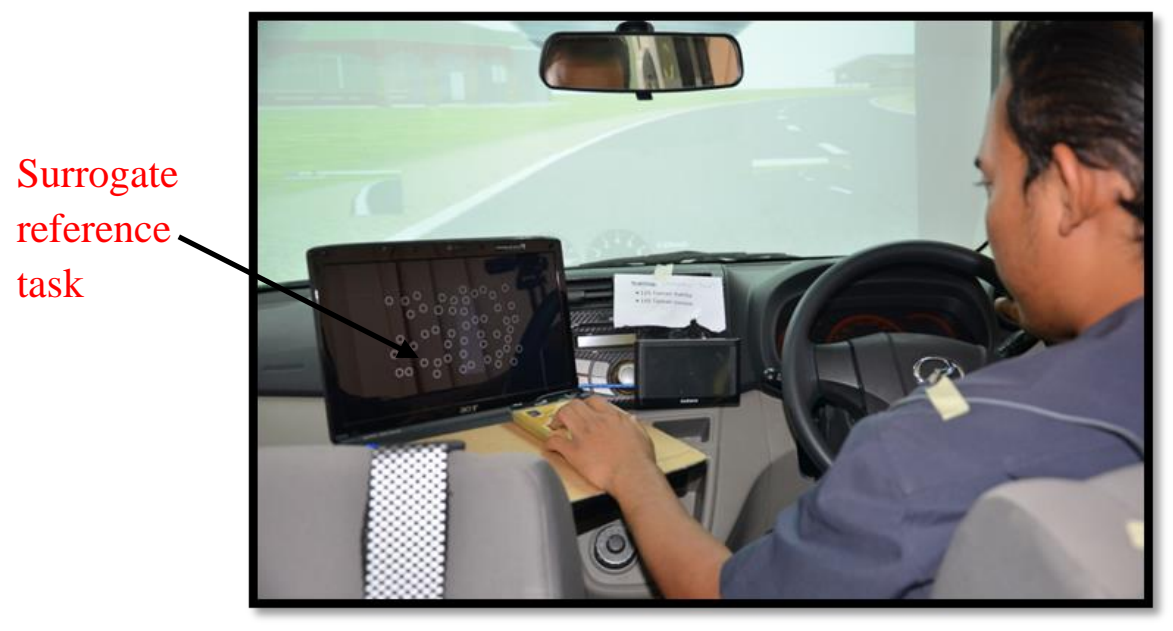

Figure 3. Surrogate reference task.

A navigation task also was employed as one of the secondary tasks. This secondary task was conducted since it is one of the sources of driving distraction [20]. An external Global Positioning System (GPS) was secured in the driving simulator using a suctioned holder designed for the system. Two addresses were provided on a sheet of paper that was secured to the dashboard. All addresses contained the same number of letters and numbers. Participants were asked to input these addresses using the touch screen of the GPS system. Texting using mobile phone also was also employed as a secondary task. This task was conducted since it is one of the main sources of driving distraction [21]. A mobile phone was placed inside the simulator. Participants were asked to reply to a series of Short Messaging Service messages using the WhatsApp application installed in the smartphones while they were driving. There were a total of three incoming messages per trial. Participants were instructed to respond to all three messages, but were not given any instructions on when to respond nor what to write in response. They were required to drive at two different speeds of 30 $\mathrm{km} / \mathrm{h}$ (low speed) and $80 \mathrm{~km} / \mathrm{h}$ (high speed).

\section{Procedure}

The implementation of the procedure required approximately one day (9.00 am to 5.00 $\mathrm{pm})$ to be completed by a participant. Before participants conducted the actual experiment session, they were required to fill out the consent form and personal details. They were given a brief overview of the experiment, its expected duration, experiment procedure, and safety. Their sickness level was also assessed before and after driving the simulator. There were 14 sub-sessions for the training session. The training session was required for the participants to familiarize themselves with each task before going in to the actual experiment session. There was no time limit given. Each training session was stopped when the participants felt comfortable and confidence. During the actual 
experiment session, each participant was required to complete the entire task and they could stop the experiment without any coercion. In each task session, participants were given approximately five to ten minutes to rest. The experiment was conducted on three different roads - expressway, off-ramp, and curved roads. When all the experiment tasks were completed, the participants were interviewed on their experience of the driving session in the specific room. Then, the participants were thanked and given consolation money for participating in the experiment.

\section{Data Analysis}

The results obtained were analysed using Analysis of Variance (ANOVA) with post-hoc tests using the Least Significant Difference (LSD) method to compare the means of three categories of road types and secondary tasks. All the statistical analyses were performed using Statistical Package for Social Science (SPSS) version 16.0. Significance levels are reported at $\mathrm{p}<0.05, \mathrm{p}<0.01$, and $\mathrm{p}<0.001$ in each respective graph.

\section{RESULTS AND DISCUSSION}

\section{Road Segment}

As illustrated in Figure 4, the same pattern of response time across the three road segments was displayed for both detection response task versions. The response time differed significantly across the three road sections for both tactile $(F(2,87)=7.33, p=$ $0.001)$ and head-mounted tasks $(F(2,87)=3.74, p=0.028)$. LSD post-hoc comparisons of the three road segments indicate that the off-ramp recorded significantly higher response time than the expressway for both detection response tasks and the curved road for tactile type only. This shows that driving in more challenging road segments requires excessive cognitive demand. The strong sensitivity to environment complexity is aligned with the studies conducted by Engstrom et al. [22] and van Winsum et al. [17], although these studies assessed different level of road segmentscity driving, expressway, and rural roads.

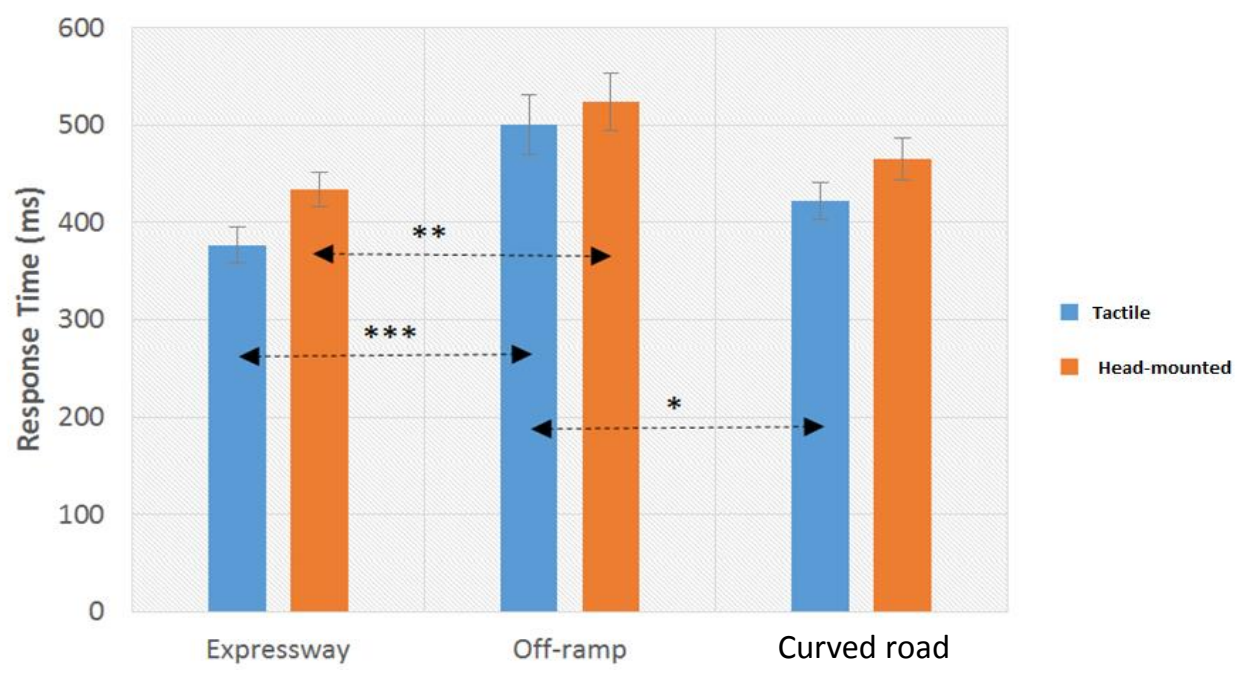

igure 4. Response times for expressway, off-ramp, and curved road segments (mean \pm standard error). 


\section{Secondary tasks for tactile type}

There was a significant effect of response time in the secondary tasks $(F(7,272)=$ 12.46, $p<0.001$ ). As illustrated in Figure 5, longer response times were observed for all secondary tasks when compared with the baseline. Only the difficult task for 1-Back was found to be significantly longer than the easier task (0-Back). This can be generally explained by the fact that 1-back has a greater working memory load than 0-back [13]. No other comparisons were significant.

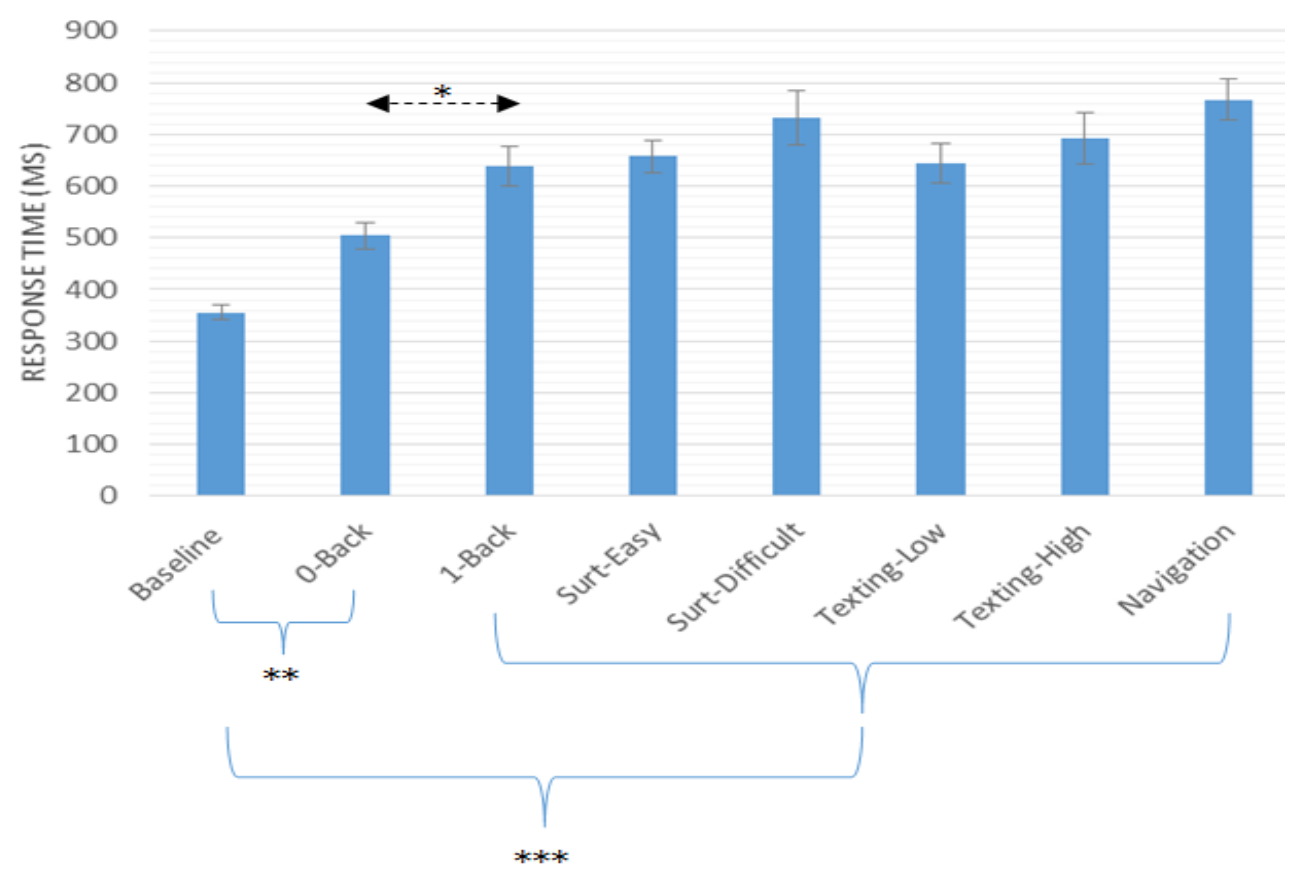

Figure 5. Response times of secondary tasks for tactile type (mean \pm standard error).

\section{Secondary tasks for head-mounted type}

Analysis using ANOVA revealed that the response time differed significantly across the secondary tasks $(F(2,272)=14.2, p<0.001)$. Further post-hoc comparisons using the LSD revealed that response times were significantly longer than baseline when any additional task was performed while driving, as indicated in Figure 6. Furthermore, in terms of the number recall task, response times were significantly longer for the more difficult 1-back task than those for the 0-back task. Comparisons between two levels of difficulty for the surrogate reference task and texting using mobile phone task showed no significance $(\mathrm{p}>0.05)$. This is consistent with the findings from multisite ISOcoordinated studies not only for head-mounted type but also for tactile type [13].

Generally, when comparing between both DRT types for all tasks, lower response time - that is, faster responses-were recorded for tactile type than headmounted type. This was expected as tactile uses tactile sense that inherently has a faster response time than head-mounted type that uses visual sense; this is consistent with previous studies [19]. Furthermore, both DRT types that are mainly intended to measure effects of cognitive load of a secondary task on attention have been validated through multisite ISO-coordinated studies [13]. 


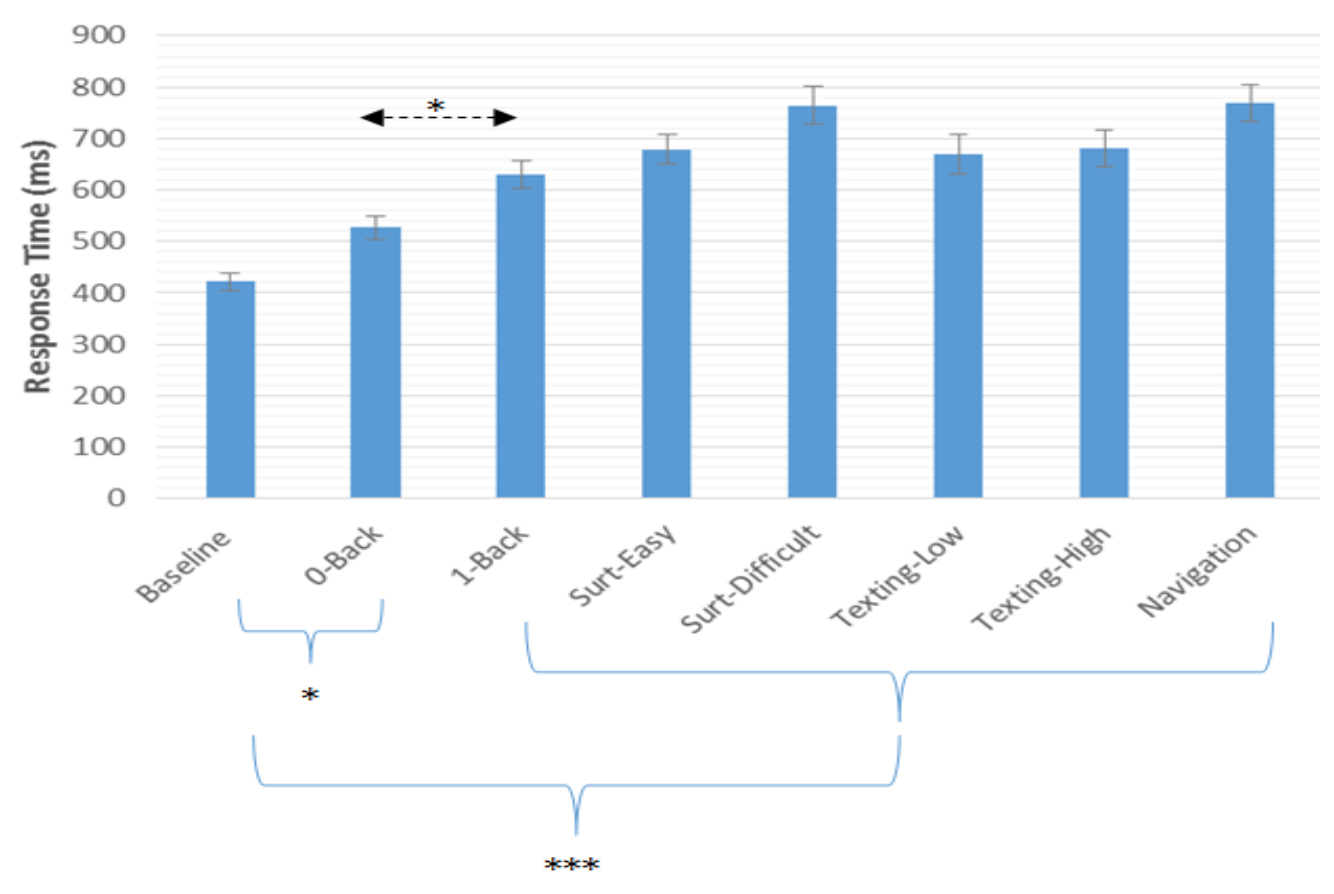

Figure 6. Response times of secondary tasks for head-mounted type (mean \pm standard error).

\section{CONCLUSIONS}

In summary, the results reveal that response times generally increased as a function of road segments as well as exposure to secondary tasks. The findings obtained from this study can provide recommendations for the working group committee ISO/TC22/SC13/WG8 in finalizing the final ISO 17488 document. In addition, it can also be used as part of a public awareness campaign on driver distraction activities, particularly on issues related to texting while driving and interaction with an In-Vehicle Information System (IVIS). As a way forward, we propose to expand the existing DRT assessment to other secondary tasks that may distract drivers during driving and explore the feasibility of utilizing the DRT for motorcycle riding in a simulated environment.

\section{ACKNOWLEDGEMENTS}

The authors would like to acknowledge Malaysian Institute of Road Safety Research for providing financial assistance under project no. RU101021. Besides, sincere gratitude to DRT task force committee under the ISO/TC22/SC13/WG8 for their full cooperation.

\section{REFERENCES}

[1] RMP. Statistical report of road accident in Malaysia. In: Police RM, editor. Kuala Lumpur: Traffic Branch, Bukit Aman; 2013.

[2] Cameron M. World report on road traffic injury prevention. BMJ Publishing Group Ltd; 2004. 
[3] Chan M, Partouche D, Pasquier M. An intelligent driving system for automatically anticipating and negotiating road curves. IEEE International Conference on Intelligent Robots and Systems. 2007; 117-22.

[4] Horberry T, Anderson J, Regan MA, Triggs TJ, Brown J. Driver distraction: The effects of concurrent in-vehicle tasks, road environment complexity and age on driving performance. Accident Analysis \& Prevention. 2006;38:185-91.

[5] McEvoy SP, Stevenson MR, Woodward M. The prevalence of, and factors associated with, serious crashes involving a distracting activity. Accident Analysis \& Prevention. 2007;39:475-82.

[6] Klauer SG, Dingus TA, Neale VL, Sudweeks JD, Ramsey DJ. The impact of driver inattention on near-crash/crash risk: An analysis using the 100-car naturalistic driving study data. US Department of Transportation: National Highway Traffic Safety Administration; 2006.

[7] Young KL, Salmon PM. Examining the relationship between driver distraction and driving errors: A discussion of theory, studies and methods. Safety Science. 2012;50:165-74.

[8] Salleh I, Md. Zain MZ, Raja Hamzah RI. Evaluation of annoyance and suitability of a back-up warning sound for electric vehicles. International Journal of Automotive and Mechanical Engineering. 2013;8:1267-77.

[9] Kang HS, Jalil MKA, Musa M. Development of a virtual driving simulator for transportation research. 1st International Conference on Product Design and Development, Sabah, Malaysia; 2004.

[10] Machmudi Isa IYA, Zainul Abidin MA, Mansor S. Objective driveability: Integration of vehicle behavior and subjective feeling into objective assessments. Journal of Mechanical Engineering and Sciences. 2014;6:782-92.

[11] Salwani MS, Sahari BB, Ali A, Nuraini AA. The effect of automotive side member filling on car frontal impact performance. Journal of Mechanical Engineering and Sciences. 2014;6:873-80.

[12] Nilsson L. Behavioral research in an advanced driving simulator-experience of the VTI system. Human Factors and Ergonomics Society 37th Annual Meeting; 1993: 612-6.

[13] Road vehicles. Transport information and control systems - Detection-Response Task (DRT) for assessing attentional effects of cognitive load in driving. New Work Item: ISO/TC 22/SC 13/WG 08 (Draft International Standard; 2015.

[14] Young K, Regan M, Hammer M. Driver distraction: a review of the literature. Victoria, Australia: Monash University Accident Research Centre; 2003.

[15] Engström J, Larsson P, Larsson C. Comparison of static and driving simulator venues for the tactile detection response task. 7th International Driving Symposium on Human Factors in Driver Assessment, Training, and Vehicle Design; 2013: 1-7.

[16] Merat N, Jamson AH. The effect of stimulus modality on signal detection: Implications for assessing the safety of in-vehicle technology. Human Factors: The Journal of the Human Factors and Ergonomics Society. 2008;50:145-58.

[17] van Winsum W, Martens M, Herland L. The effects of speech versus tactile driver support messages on workload driver behaviour and user acceptance: TNO-Report, TM-99-C043: TNO Human Factors Research Institute; 1999.

[18] Mehler B, Reimer B, Dusek J. MIT AgeLab delayed digit recall task (n-back). Cambridge, MA: Massachusetts Institute of Technology. 2011. 
[19] Mattes S, Föhl U, Schindhelm R. Empirical comparison of methods for off-line workload measurement. AIDE Deliverable. 2007;2.

[20] Ranney TA. Driver distraction: A review of the current state-of-knowledge. U.S. Department of Transportation: National Highway Traffic Safety Administration; 2008.

[21] Cheung D. Text messaging while driving: its effects on driving performance and text messaging behaviour. Master of Science (Human-Computer Interaction with Ergonomics), Faculty of Life Sciences, University College London; 2010.

[22] Engström J, Åberg N, Johansson E, Hammarbäck J. Comparison between visual and tactile signal detection tasks applied to the safety assessment of in-vehicle information systems. Proceedings of the Third International Driving Symposium on Human Factors in Driver Assessment, Training and Vehicle Design: Citeseer; 2005: 232-9. 